\title{
¿Cómo narrar la realidad económica? Discusión de los marcos y los encuadramientos cognitivos en economía.
}

Camilo Andrés Guevara Castañeda

Universidad Nacional de Colombia 


\title{
¿Cómo narrar la realidad económica? Discusión de los marcos y los encuadramientos cognitivos en economía.
}

\author{
Camilo Andrés Guevara Castañeda ${ }^{1}(\mathbb{D})$ \\ Universidad Nacional de Colombia-Colombia
}

\section{RESUMEN}

Este artículo pretende aportar a la conversación sobre los encuadramientos mentales y al debate reciente sobre epistemología en economía. Por otro lado, y a diferencia de propuestas como la de Tony Lawson, quien pretende mostrar que el punto central de las divergencias entre el análisis ortodoxo y heterodoxo en economía se debe al tipo de ontología que se asume, intentamos mostrar que un elemento central de esta divergencia se encuentra en los encuadramientos cognitivos con los que se discute. Se han escogido algunos casos teóricos y de análisis en los que sé que muestran como los "hechos" son descritos con relación a un marco cognitivo, lo que señala las diferencias en los puntos de partida al analizar la realidad. El objetivo está en entender que hablamos dentro de marcos cognitivos que nos condicionan y condicionan las discusiones que damos, y que generalmente terminan invisibilizando otras maneras de mirar, de plantear preguntas o de iniciar discusiones.

Palabras clave: Marcos cognitivos; Epistemología de la economía; Heterodoxia; Equilibrio; Racionalidad.

JEL: B31, B40, B50.

\section{How to narrate the economic reality? Discussion of cognitive frameworks in economics}

\section{ABSTRACT}

This article aims to contribute to the conversation about frameworks and the epistemology of economics. On the other hand, and unlike proposals such as Tony Lawson's, which aims to show that the central point of the differences between orthodox and heterodox economics is due to the type of ontology that is assumed, we try to show that a central element of this divergence is found in the frameworks. Some theoretical cases have been chosen in which we show how the "facts" are described in relation to a cognitive framework, which indicates the differences in the starting points when analyzing reality. The objective is to understand how frameworks determine the economic discussions.

Keywords: Cognitive frameworks; Epistemology of economics; Heterodoxy; Balance; Rationality. JEL: B31, B40, B50.

\footnotetext{
${ }^{1}$ Candidato a Doctor en Ciencias Económicas. Profesor de Economía de la Pontificia Universidad Javeriana y de la Universidad Nacional de Colombia.
} 


\section{INTRODUCCIÓN}

En nuestra vida cotidiana cuando nombramos y describimos algún fenómeno o estado de cosas, tenemos la convicción de estar dando cuenta de su significado intrínseco. En otras palabras, de la manera de ser que el fenómeno tendría en sí mismo, incluso si nadie lo observara. Esta visión ha sido desafiada de múltiples formas, sin embargo, una de las versiones más interesantes de este desafío ha sido la desarrollada por el lingüista cognitivo George Lakoff, quien nos muestra como la manera en que entendemos los fenómenos está guiada por campos metafóricos y encuadramientos cognitivos (Lakoff, 2009). En ese sentido, lo que decimos, pensamos y hacemos está impregnado por marcos que trazan un horizonte de sentido. Allí radica su pertenencia, en la trama de significado que crean y las consecuencias que se derivan de los diferentes horizontes creados. Por supuesto, esto no quiere decir que los marcos no se vean alterados por la experiencia o que haya solo un vínculo unidireccional entre nuestra experiencia y los marcos cognitivos. Más bien, la experiencia y los campos se influencian conjuntamente.

Lakoff nos ha mostrado el impacto que tienen los encuadramientos y los marcos cognitivos, desde un caso corriente como la forma en la que abordamos una discusión (Lakoff, 2009), hasta las campañas políticas (Lakoff, 2008). En el primer caso, generalmente se vive una discusión como un escenario de guerra en el que se trata de ganarle al adversario. No obstante, según Lakoff, este marco no es intrínseco a la discusión. Podríamos también discutir como si estuviéramos danzando y tratando de bailar con los argumentos del adversario. Si el marco de discusión fuera una danza, no se adornaría solamente un "hecho" (el discutir) con pinceladas de amabilidad. No solo cambiaría el tono emotivo de la conversación, sino que cambiaría la raison d'être de una discusión. Estamos en presencia de otro acontecimiento. Entre otras cosas, una danza no posibilita la violencia física, no está en su horizonte de sentido, como si sucede con algunas discusiones cotidianas, que terminan en ella.

En el segundo caso, Lakoff también ha usado su teoría para mostrarnos como los encuadramientos que utilizan las campañas políticas son esenciales para lograr llegar a los electores. Por ejemplo, los conservadores en Estado Unidos, ligados al partido republicano, recurren a la concepción del padre estricto en lugar del padre protector. Esto tiene consecuencias porque el encuadramiento del padre estricto guía todo a los actos individuales. Si el individuo es pobre es porque no ha sido suficientemente disciplinado. Se moldean entonces los problemas bajo el esquema de un padre con autoridad que sanciona a sus hijos cuando no son responsables. El éxito y el fracaso son así problemas morales. Los problemas se resuelven individualmente. Es el ejemplo de "los conservadores que creen que los desempleados han optado por no trabajar, así el misterio profundo de la infelicidad de los desempleados es más un problema de los psicólogos más que de los economistas." (Stiglitz, Ocampo, Pfrench-Davis, Spiegel, \& Nayyar, 2006). Se trata de encontrar, según la celebre expresión del sociologo aleman Ulrich Beck, de encontrar "soluciones biográficas a contradicciones sistemicas" (Beck \& Beck-Gernsheim, 2003, pág. 31).

En economía, poco solemos prestar atención al problema de los encuadres cognitivos. Se piensa que esto no hace parte del núcleo de la disciplina y por lo tanto que son otras disciplinas las que deben reflexionar sobre estos temas. En el fondo, esta actitud refleja 
una falla fundamental en la formación de los economistas quienes terminan creyendo después de su formación que la reflexión filosófica, los cursos sobre filosofía de la ciencia y la epistemología son una pérdida de tiempo. No obstante, estas reflexiones son fundamentales, pues apuntan sobre todo a mostrar que nuestros criterios de valoración podrían ser otros.

Una de las pocas reflexiones en economía, que recoge la perspectiva de los encuadramientos mentales, ha sido la de la profesora Kate Raworth de la Universidad de Oxford, que en su libro Doughnut Economics (2017) señala la importancia de "redibujar" la economía para tratar de incrustarla en sus raíces medioambientales y sociales. De esta incrustación (embedded), surge un gráfico de una rosquilla o dona, que señala el espacio de posibilidad para poder "satisfacer los derechos humanos de cada persona y dentro de los medios de nuestro planeta dador de vida" (Raworth, 2017). Este gráfico pretende desafiar la representación del diagrama de flujo circular que se enseña en las primeras clases de introducción a la economía y que fue diseñado originalmente por Paul Samuelson en su manual de 1948 (Samuelson, 1957). Este gráfico continúa ejerciendo influencia de lo que es relevante en el proceso económico. El diagrama de flujo circular muestra que lo más importante es el flujo de las mercancías y de dinero entre familias y empresas, como si de un trueque se tratara. El esfuerzo de Raworth va dirigido a cambiar el marco o referente que tenemos para pensar la economía. Así, pretende cambiar la manera en que conversamos.

Este artículo pretende aportar a la conversación de la importancia de los encuadramientos mentales y de las maneras de conversar y relatar los hechos. Por otro lado, y a diferencia de propuestas como la de Tony Lawson (1997), quien pretende mostrar que el punto central de las divergencias entre el análisis ortodoxo y heterodoxo en economía se debe al tipo de ontología que se asume, intentamos mostrar que un elemento central de esta divergencia se encuentra precisamente en los encuadramientos mentales en los que se discute.

Se han escogido algunos casos que muestran como los "hechos" son descritos con relación a un encuadramiento mental, lo que muestra también las diferencias en los puntos de partida al analizar la realidad. Seguimos entonces la estrategia de oponer a la lectura habitual y dominante de los hechos otro encuadre, sin dejar de lado que podrían existir otras posibilidades de encuadramiento. Por supuesto, al querer mostrar la pertinencia de los encuadramientos cognitivos en diferentes tópicos económicos, no se pretenden abordar exhaustivamente los mismos. El objetivo está en entender que hablamos dentro de marcos cognitivos que nos condicionan y condicionan las discusiones que damos, y que generalmente terminan invisibilizando otras maneras de mirar, de plantear preguntas o de iniciar conversaciones.

El articulo está organizado de la siguiente forma. En la sección dos se analiza el encuadramiento cognitivo en el que se dan las discusiones sobre el funcionamiento de los mercados y se señala como pueden leerse los fenómenos desde el desequilibrio. En la tercera, se aborda el problema del encuadramiento en la noción de racionalidad y los sesgos cognitivos. En la cuarta se analiza el encuadramiento que ha dado la economía hacia la información en detrimento del conocimiento. En la quinta sección se retoman las discusiones contemporáneas sobre el neoliberalismo y su definición como racionalidad y 
a ella se opone otro encuadramiento mental (lo común). Por último, en la sexta se problematiza el binomio formal/informal para leer la realidad laboral y se muestra una lectura desde la economía popular que se aleja de esta visión dual. El capítulo cierra con una reflexión sobre la importancia de los marcos cognitivos en los diferentes temas tratado y su importancia a la hora de narrar la realidad y no caer en el juego del marco que se quiere combatir.

\section{Mercados en equilibrio y fallos de mercados versus mercados endógenamente inestables}

Como se sabe, en los años cincuenta del siglo pasado, Kenneth Arrow y Gerard Debreu ofrecieron una prueba matemática a la inquietud de Adam Smith sobre si una economía con agentes descentralizados tiene algún mecanismo de coordinación, de modo que los intereses y las acciones de los mismos no generen un caos. Ellos demostraron que bajo ciertos supuestos existe al menos un vector de precios de equilibrio que vacía los mercados (Arrow \& Debreu, 1954). Aunque demostraron la existencia del equilibrio, posteriormente vinieron las dificultades para mostrar la unicidad y estabilidad del mismo. La prueba de la existencia del equilibrio tuvo una mayor trascendencia, que los teoremas que mostraban las condiciones extremas bajo las cuales la unicidad y estabilidad del equilibrio se conseguían (Teoremas de Sonnenschein, Mantel y Debreu (SMD) (Sonnenschein, 1972); (Mantel, 1974). Sin duda, la prueba de la existencia del equilibrio ha ayudado a confirmar la confianza de la mayor parte de economistas en la justificación del mecanismo de mercado, que han tenido desde que su representación subyace a una economía de trueque, y así justificarlo como un óptimo asignador de recursos. Aunque el modelo A-D tenía que asumir supuestos irreales (no hay externalidades, hay competencia perfecta, existen mercados para todo, no hay bienes públicos, etc.), se justificó en un primer momento bajo el paraguas del instrumentalismo neoclásico, al que Milton Friedman dio su mayor justificación al comparar la física con la economía y sugerir que la metodología de la economía positiva estaba orientada a la predicción y no a la descripción de los hechos reales (Friedman, 1966). El instrumentalismo ha servido para la justificación de muchos modelos al diluir la crítica de equiparar la realidad al modelo (la crítica de que el modelo no es realista). Así, desde esta interpretación el modelo ArrowDebreu no era una representación de una economía capitalista sino una economía imaginaria cuya utilidad era generar predicciones. Sin embargo, esta justificación de la teoría no ha dejado de generar problemas, pues al cambiar un supuesto se puede llegar a una conclusión diferente y por lo tanto apoyar una política o una realidad que se desea justificar. En resumen, el modelo es sensible a los supuestos que pueden ser utilizados para justificar agendas políticas. En nuestros días, algunos como (Rodrick, 2016) sostienen un instrumentalismo perfeccionado que trata de responder a esta crítica. Se argumenta que se pueden sostener supuestos irreales desde que no afecten las conclusiones del modelo. En otros términos, los supuestos críticos, aquellos que son claves para el modelo y que al alterarlos también cambian los resultados del modelo, no pueden ser irreales.

Sin embargo, algunos neoclásicos no estuvieron de acuerdo con la justificación de Friedman, entre ellos Paul Samuelson y Kenneth Arrow. Este último ha argumentado a favor de juzgar la pertinencia del modelo por su distancia a la realidad. Si la realidad no se comporta como el modelo entonces hay una brecha entre las dos; una falla de la realidad. Así hoy se da mayor preponderancia a las externalidades, la asimetría de información, la existencia de bienes públicos, la no existencia de mercados completos, los 
monopolios etc. las cuales permiten juzgar la realidad desde una norma. El vocablo de las fallas de mercado está hoy enraizado en el sentido común de economistas y políticos y ha abierto una caja de herramientas que dicta su hacer a los economistas en la que estos como ingenieros deben diagnosticar una falla y corregirla.

Sin embargo, es necesario hacer una operación de desnaturalización. Cuando hoy se habla de fallos de mercado, lejos de verse como una descripción que es posible por un marco cognitivo, es decir que el fallo refiere a un orden, en este caso a un mercado perfecto, se ve como una descripción del modo de ser intrínseco de la realidad. Esto ha llevado al punto en que algunos economistas que incluso eran críticos con la aproximación instrumental neoclásica, hoy utilicen conceptos como el de fallos de mercado. Así, bajo estas coordenadas la idea de equilibrio sigue permeando nuestra manera de conocer en economía, y como dice Lakoff, si sigues discutiendo en el marco del contradictor pronto vas a perder el control de la discusión.

Aunque alguien podría reclamar que las investigaciones sobre el modelo A-D han declinado y que la economía ahora tiene otras agendas y técnicas de investigación, se puede ver que la idea de equilibrio sigue permeando aspectos fundamentales del pensamiento macroeconómico y sus recomendaciones de política. Es el caso de la política monetaria, que, a través de la regla de Taylor, que opera al reducir las brechas del producto y la inflación, y con conceptos como la tasa natural de interés, activa una visión de una economía en equilibrio. Se trata de una herramienta que permite a los bancos centrales elegir el valor de la tasa de interés nominal que llevaría a la economía a ese estado imaginario del equilibrio. Se traza un horizonte de sentido a la realidad, o en el lenguaje de Lakoff, un marco cognitivo, en lugar de simplemente estar describiendo los hechos y estar dando cuenta de lo que son las cosas.

\section{Desequilibrio}

En su obra John Maynard Keynes, el economista Hyman Minsky observó que uno de los elementos centrales de la Teoría General de Keynes y olvidada por las reconstrucciones neoclásicas, es que nuestra vida no se lleva a cabo en situaciones de equilibrio, sino en las transiciones hacía estas, sin alcanzar jamás estos puntos de equilibrio (Minsky, 1990). Podríamos ver un ejemplo cotidiano cuando entramos a una tienda de ropa que está en rebajas (sales) y preguntamos el costo de una prenda que nos gusta y nos dicen que ese producto no tiene descuento pues es de nueva colección. Esto significa que antes de haber vendido toda la colección anterior; vaciar el mercado o haber alcanzado un equilibrio, ya estamos dentro de un nuevo periodo de producción.

Según esta interpretación, el núcleo central de la obra de Keynes no es que el problema sean los fallos de mercado, como cierta interpretación New Keynesian trata de posicionar por medio de recursos teóricos que dan cuenta de las rigideces reales o nominales en los mercados de trabajo, bienes o crédito, como los costos de ajuste de los precios, la competencia imperfecta, los salarios pegajosos o las fallas de coordinación, entre otros (Mankiw \& Romer, 1991). El problema desde este marco cognitivo es el mismo libre mercado que en sí mismo no puede llevar a una economía al empleo de todos sus recursos. 
Sobre este camino, Minsky realizo sus propias investigaciones sobre la dinámica financiera de las economías capitalistas, que en la perspectiva tradicional se entiende como un carburador, aquel que organiza la mezcla de aire y combustible (Ahorro e inversión) y permite liberar "energía" para lograr el crecimiento económico, lo que lo supone neutral al proceso de mercado. Minsky, bajo la tutela de su maestro Schumpeter, entendió tempranamente que el proceso de otorgar créditos no es principalmente un proceso de transferencia del poder de compra de alguien que tiene excedentes a otro que no tiene ${ }^{2}$, sino que es fundamentalmente un proceso de creación de un poder de compra adicional, que distorsionan la economía, más cuando se pasa de un esquema en el que las utilidades alcanzan para pagar el principal y los interés del crédito a un esquema Ponzi en el que se piden nuevos créditos para pagar los interés de la deuda (Minsky, 1990). No solo entonces los diferentes comportamientos y preferencias hacen que el mercado sea inestable (vía la dificultad de lograr una curva de demanda con pendiente negativa del mercado y por lo tanto logrando múltiples equilibrios) sino que la existencia del dinero, entendido como deuda, también distorsiona el mercado. El mercado entonces que es eficiente de Pareto, como muestran los teoremas del bienestar de los manuales de texto, es el de una economía de trueque y simplificada, pero no el de una economía monetaria de producción. Las crisis financieras recurrentes son solo una muestra de su inestabilidad.

Tenemos entonces otro marco cognitivo que relaciona lo real respecto a una norma de desequilibrio. Este marco posibilita otros modos de ser. Otras respuestas para la pregunta ¿Para qué sirve el Estado en una economía capitalista? Mientras en el marco cognitivo de equilibrio, la versión extrema (New Classical) sostiene que el mercado es esencialmente estable, que solo puede ser perturbado por fuerzas externas (choques de productividad, cambio tecnológico, sindicatos, etc.) y los individuos con sus capacidades pueden actuar volviendo a una senda de equilibrio. La versión "menos radical" (New Keynesians), sostiene también que el mercado es esencialmente estable pero debido a ciertas rigideces los mercados no pueden vaciarse en el corto plazo. Esto abre entonces la puerta a que el Estado pueda corregir ciertas fallas de mercado, aunque desde que se pueda se prefiere que puedan ser corregidas vía solución de mercado. En resumen, el Estado o ve anulada su capacidad de actuación por fuerzas superiores a él o solo debe corregir las fallas de mercado, limitándose a aceptar la asignación de recursos ejercida por él y ejecutando programas que no distorsionen esa asignación primaria del mercado. En otras palabras, el Estado en lugar de establecer las reglas de juego y comandar el mercado, debe supeditarse a él. No obstante, como anota Mariana Mazzucato en su reciente libro The Value Of Everything, la creación de Internet, el GPS, las pantallas de cristal líquido (LCD), entre otros, no hubieran sido creados si el Estado solo se hubiera dedicado a respetar las asignaciones de mercado. No se hubiera enviado un hombre a la luna si se hubiera pensado que el rol del Estado era solo corregir las fallas de mercado (Mazzucato, 2016). Se tuvo ante todo un propósito, una intencionalidad. Esto por supuesto abre otro entendimiento del para qué del estado. Hoy por ejemplo ante grandes desafíos como el cambio estructural a energías limpias. El estado podría ir más allá de solo cobrar impuestos sobre el carbono, y ejercer más activamente un rol en esta transformación, teniendo o comprando activos estratégicos que potencien la generación de energías limpias e incentivando o configurando los mercados para su transición.

\footnotetext{
${ }^{2}$ Con lo cual no existirán distorsiones en el mercado o solo existirían perturbaciones mínimas si hay cambios extremos
} en la propensión marginal a consumir. 
En resumen. Bajo el marco cognitivo del desequilibrio, tenemos que al Estado se le abren otros mundos de lo posible, de lo que se puede debe hacer. En primer lugar, un gobierno grande estabiliza una economía que es de por sí inestable (Minsky, 1982) No solo se trata de los llamados estabilizadores automáticos, lo cual supone una visión defensiva del Estado, sino que se justifica que el Estado pueda intervenir reasignando la asignación de los mercados pues estos no suelen ser eficientes. Los equilibrios de una economía monetaria, a diferencia de una de trueque, no son óptimos de Pareto (Starr, 2013). Se justifican plenamente los subsidios a la oferta, y en general, las intervenciones que tienden a desmercantilizar y establecer otros horizontes más allá de la competencia, pues el mercado en sí mismo es inestable o distorsionado y estas intervenciones lo estabilizan entre otras cosas al aumentar el ingreso disponible de las personas. Por último, se justifican las intervenciones de gran envergadura, en las que hay grandes propósitos y en las que el sector privado solo suele ver costos (Mazzucato, 2014).

\section{Sesgos cognitivos o racionalidad para mortales}

Del mismo modo que en la sección anterior establecimos que solo es posible hablar de fallos de mercado con referencia a una norma (no pueden existir fallos sin un orden o norma definida previamente, todo fallo es fallo de algo). En esta sección mostramos que la llamada irracionalidad y los sesgos cognitivos son descritos tomando distancia respecto a una noción de racionalidad. En otros términos, la economía comportamental, aunque reclama mayor empirismo frente a la hiperracionalidad asumida tradicionalmente en los modelos económicos, juzga lo real por la distancia que existe entre el comportamiento ideal y lo que encuentra como real. Pero ¿Cuál es esa norma ideal?

El estudio de las características que involucran la toma de decisiones de los agentes en su cotidianidad se mostró para los iniciadores del proyecto Walrasiano como un terreno gelatinoso, que involucraba los deseos, las creencias, los temores etc. La respuesta entonces fue postular la idea de que los individuos orientan la toma de decisiones de forma "racional" y universal, generando así respuestas homogéneas a las diferentes situaciones.

La teoría neoclásica de la racionalidad visualiza el problema económico à la Robbins, es decir como un problema de relación entre unos fines psicológicos y unos recursos que son escasos. Se trata de realizar elecciones que deben maximizar la utilidad individual.

Esta teoría día un paso más allá cuando dio cuenta de la toma de decisiones cuando no se conocen a priori los resultados. Esto es el caso por ejemplo de las loterías. Se construyó así lo que se conoce como la teoría de la utilidad esperada, que axiomatizaron Von Neuman y Morgenstern. Esta teoría es una construcción "en torno a un conjunto específico de preceptos lógicos (Integridad, transitividad, independencia y continuidad) formulados para producir ordenamientos de preferencias bien definidas" (Davis, 2011). Así, la hiperracionalidad necesita primero que las preferencias sean coherentes, consistentes y bien definidas, y que, dado un conjunto de opciones de elección, el individuo maximice su función de utilidad. De esta forma los individuos están bien capacitados para obtener los mejores resultados posibles, al guiarse por sus propios intereses. A su vez, dado que el mercado proporciona la información y los incentivos 
pertinentes, al maximizar la utilidad individual también se maximiza el bienestar social entendido como la suma de las utilidades individuales (Sen, 1977).

Esta forma de plantear el problema de la racionalidad rechaza cualquier relacionamiento con la psicología y la filosofía, disciplinas que se ven con desconfianza. Se trata así de explicitar los procesos que subyacen a los fenómenos mentales por medio de la lógica y los formalismos matemáticos. Se sostiene así que "la lógica es la disciplina adecuada para comprender el cerebro y la actividad mental y que el cerebro es un dispositivo que encarna principios lógicos en sus elementos o neuronas" (Varela, 2005).

Partiendo de este impulso, se terminó por entender la cognición como computación, como procesamiento de información y símbolos que representan algo del mundo y denominado cognitivismo La llamada economía comportamental ha introducido entonces herramientas de las ciencias cognitivas, como la psicología cognitiva y la neurociencia, para tratar de construir modelos empíricos del comportamiento. Sin embargo, pretende dar cuenta de las realidades observables de los procesos de toma de decisión como divergiendo de la hiperracionalidad, a la vez que sostienen que el proceso cognitivo es el de procesamiento de información. Esta racionalidad actúa como marco cognitivo para formular los sesgos cognitivos, de la misma manera que la idea de equilibrio actúa respecto a las fallas de mercado.

Respecto a la justificación de los sesgos, Kahneman distingue dos tipos de procesos en la acción; el automático y el reflexivo. Relaciona el primero con la rapidez, la emocionalidad, la asociatividad, el procesamiento paralelo y el aprendizaje lento en la toma de las decisiones (Kahneman, 2003), que según él lleva generalmente a tomar malas decisiones. Para tomar buenas decisiones, deberíamos acercarnos al pensamiento reflexivo, lento, serial, neutral y que requiere esfuerzo. El tipo de proceso de acción que se cultiva en las universidades. Los sesgos se producen entonces porque actuamos más con el primer procesamiento que con el segundo. Los sesgos tienen así su origen en la intuición, los instintos, las emociones, la moral, las normas sociales y la cultura (Kahneman, Slovic, \& Tversky, 1982); (Pfister \& Böhm, 2008); (Wang, Simons, \& Brédart, 2001).

Entre los sesgos más famosos y utilizados en varios subcampos, como las finanzas, están los sesgos de correspondencia (infravalorar los roles, situaciones y circunstancias en la explicación de comportamientos), de confirmación (tendencia a creer aquella información que confirma las propias creencias) y retrospectivo (tendencia a creer, una vez conocemos los resultados de un proceso, que lo sabíamos desde antes) etc. Esto ha abierto un amplio campo de investigación en el que se siguen añadiendo sesgos y herramientas para corregirlos. Como dice David Primrose: "Al articular la racionalidad limitada como una "desviación" irracional del Homo Economicus, la economía comportamental posiciona al Homo Economicus como el sujeto ideal para los mercados en funcionamiento y potencialmente realizable a través de medidas correctivas" (Primrose, 2017, pág. 2). Aquí cobran importancia los denominados Nudges (Thaler \& Sunstein, 2008), que por medio de formas "no coercitivas" tratan de moldear comportamientos de la gente de acuerdo a la norma de racionalidad estándar. En otras palabras, se trata de moldear comportamientos para que la gente se comporte de manera neoclásica, lo que revela ante todo un proyecto constructivista; querer que la realidad se comporte de determinada manera. 
Otro tipo de cognición y racionalidad

A diferencia del punto de partida que entiende los fenómenos mentales como procesos lógicos formales o procesamiento de información, se tienen por lo menos otros dos puntos de partida diferentes. El primero es el conexionismo que no ve en el cerebro físico ningún procesador de información ni constituido por reglas lógicas, sino "interconexiones masivas de forma distribuida que cambian como resultado de la experiencia" (Varela, 2005, pág. 95). Esto lleva al estudio de las propiedades emergentes y el estudio de los procesos autoorganizativos como elementos claves del cerebro que no remite principalmente a la lógica. Aquí resaltan autores como Daniel Dennett y Douglas Hofstadter.

El segundo paradigma es el enactivo que como punto de partida constituye una crítica a un supuesto clave de los anteriores paradigmas (cognitivismo y conexionismo). Esto es la idea de que el conocimiento es representación de un mundo que existe independientemente del sujeto. Desde la perspectiva enactiva, "las aptitudes cognitivas están inextricablemente ligadas a una historia vivida. Tal como una senda que no existe, pero hace camino al andar" (Varela, 2005). Hay una clara circularidad entre actuar e interpretar, entre hechos e interpretaciones.

Dados estos diferentes puntos de partida, podemos plantear que hay otras normas diferentes desde las cuales se pueden conceptualizar los fenómenos mentales. Sin embargo, más que ahondar en las diferencias entre estos dos paradigmas (enactivismo y conexionismo), adoptaremos una perspectiva holística de elementos que podrían compartirse en contravía del paradigma de los sesgos cognitivos y su noción de racionalidad ${ }^{3}$.

El punto central que sirve de puente a estas visiones sobre el proceso cognitivo, es que aquello que se ve como sesgo, como una desviación o algo negativo, no tiene que serlo. Como argumenta (Gigerenzer, 2008), los proceso intuitivos e instintivos nos ayudan a tomar decisiones inteligentes. Dicho de otra manera, no es cierto que solo el análisis pausado y reflexivo nos lleve a tomar buenas decisiones. Las emociones, las razones del corazón, la cultura, los entornos etc. no son perturbadores de la racionalidad, sino que hacen parte fundamental de la vida humana y son necesarios para elegir y lo pueden hacer muy bien.

A su vez, también acordarían en sostener que algunas veces menos, es más. Esto frente al ideal de racionalidad de que siempre es preferible más a menos. Es claro que la simplicidad ayuda a tomar buenas decisiones.

En resumen, es posible dirigir las realidades observables a otro marco cognitivo, a otra norma y por consiguiente a otra forma de narrar la toma de decisiones en la vida económica.

\footnotetext{
3 Incluso desde el mismo cognitivismo se podría argumentar en contra de la racionalidad neoclásica, como lo hizo Herbert Simon y actualmente Gerd Gigerenzer. Aunque estos autores estarían de acuerdo en afirmar que las actividades mentales son fundamentalmente procesamiento de información, critican la forma en la que opera la mente para tomar buenas decisiones, pues para ellos no se trata de analizar una gran cantidad de información sino en desechar la que no se necesita (Gigerenzer, 2008).
} 


\section{Información versus conocimiento}

En la sección anterior se señaló que la cognición se consolido, desde las conferencias Macy y el surgimiento de la cibernética, como un fenómeno de procesamiento de información (Varela, 2005) ${ }^{4}$. La noción de información, cuya raíz latina remite al verbo informare, sugiere la expresión de dar forma a algo, de moldear; el significado con el que nació la palabra información remite "al acto de infusión con forma" (Mirowski \& Nik-Khah, 2017). Esta noción toma relevancia en el contexto de las ciencias de la comunicación, al tratar de entender como los canales pueden transmitir un mensaje entre un punto emisor y un punto receptor. Sin embargo, lograría tener una resonancia cultural inimaginable. Como dice Mirowski y Nik-Khah, con la noción actual de información "un verbo se reificó como un sustantivo, primero como un número y después como una cosa, y finalmente en un principio cósmico de organización alrededor del cual nuestra época gira supuestamente" (Mirowski \& Nik-Khah, 2017, pág. 45). La noción de información paso entonces de tener lugar al explicar la mente, a brindarnos una manera de entender el mercado y el rol de la sociedad y las personas.

Así como los walrasianos adoptaron el cálculo infinitesimal en la economía, al ser influenciados por lo que se estaba desarrollando en otras disciplinas y la posibilidad de despolitizar la economía, Friedrich Hayek, quien estaba al tanto de lo que sucedía en otras disciplinas, fue el primero que trajo el paradigma informacional a la economía para reconfigurar el debate sobre la superioridad de una economía de mercado sobre una planificada. Esto es la controversia sobre la posibilidad del cálculo racional en una economía socialista, en la que el argumento de Mises, según el cual este cálculo era imposible en el socialismo y por tanto su inferioridad, había sido derrotado por economistas como Oskar Lange. Así, Hayek logro cambiar el marco cognitivo sobre el cuál se pensaba el mercado. Ya no era un dispositivo mecánico bajo el cual se interpretaban las leyes de oferta y demanda, sino ante todo se estaba en presencia de un gran procesador de información fruto de un orden espontaneo (ni natural, ni diseñado). Esto cambiaba la conversación, o los términos de la misma, para justificar la superioridad del mercado. Curiosamente, los economistas de la Cowles Foundation, entre los que se encontraba Hurwicz y Arrow entre otros, y que a finales de los años 40 se denominaban socialistas de mercado (Mirowski \& Nik-Khah, 2017), intentaron rebatir la proposición de Hayek, en su propio terreno. No obstante, un proyecto que esperaba establecer un mecanismo alternativo de asignación de no-mercado, pero "parecido a un mercado", empezó a aceptar la distinción entre mecanismos centralizados y descentralizados. El diseño de mecanismos de Hurwicz fue inicialmente pensado como un proceso de ajuste diferente al tradicional del mercado, pero que pudiera mejorar el rendimiento del mercado sin imponer muchos requerimientos comunicacionales. Pero una vez en el terreno de la información, se hizo evidente el rechazo a los mecanismos centralizados. Por esas ironías de la historia, la Cowles foundation terminaría fortaleciendo aún más el paradigma informacional y la posición de Hayek.

En la trayectoria que ha seguido la economía informacional, se sigue teorizando la información de tres maneras. Como una mercancía, como un índice inductivo y como computación simbólica (Mirowski \& Nik-Khah, 2017). Estas concepciones cambian la

4 Por supuesto también había algunas voces disidentes como Gregory Bateson (Varela, 2005) 
manera en que se genera la información, el tiempo en el que se genera y la importancia del proceso cognitivo entre otros ${ }^{5}$. La concepción de información va a definir que son y que hacen los mercados. Esto es de tremenda importancia, pues el paradigma informacional va a debilitar la idea del mercado como entidad universal que puede ser criticada en términos generales. Se tienen diferentes mercados, que hacen diferentes cosas. Uno de los aportes interesantes del libro de Mirowski y Nik-Khah es que estos autores relacionan las concepciones de la información, con los diferentes roles que asume el sujeto cognoscente, en particular con la evolución de las ideas de Hayek sobre el sujeto cognoscente.

En un primer momento, la concepción de Hayek ve el conocimiento como disperso en la sociedad, y lo que hace el mercado es procesar esta información dispersa. Sin embargo, en esta etapa el conocimiento es importante. Esta concepción del conocimiento como disperso pero importante, se corresponde con la noción de información como mercancía. En una segunda etapa, Hayek ve el conocimiento como "tácito e inaccesible a la auto examinación", este se corresponde con la idea de la información como índice inductivo ${ }^{6}$. El proceso cognitivo tiene lugar entonces como inferencia estadística, pero el sujeto no puede lograr una plena autocomprensión ni auto explicación del conocimiento. La última concepción de Hayek es que la cognición es irrelevante y se corresponde con la noción de información como computación y según Mirowski y Nik-Khah encarnada por la economía experimental. Los autores muestran como los experimentos de Vernon Smith, el creador de la economía experimental, se originaron en refutar los experimentos de Chamberlin que mostraban que la libre competencia no generaba resultados eficientes. Smith entonces se propuso demostrar como en presencia de una doble subasta, dado unos precios y cantidades y en ausencia de cualquier conocimiento, la oferta y la demanda lograban rápidamente converger a un equilibrio. Smith, llamo "Hipótesis de Hayek" a la idea de que los individuos en el mercado necesitan poca o nula información para llegar a decisiones correctas, pues el sistema de precios es un gran procesador de información.

El punto clave es que el paradigma informacional ha redefinido los mercados, su razón de ser y lo que hacen, y con esto desplazan el lugar que el mismo ser humano ocupa en la sociedad. Si antes se discutía sobre si los mercados eran el mecanismo que nos llevaba de manera más eficiente a lo que queríamos, era claro que se discutía sobre la base de que los individuos decidían que querían. El marco cognitivo informacional desplaza esta cuestión, pues representa la vida social mostrando que los individuos no saben lo que quieren, pero eso no importa porque los mercados si lo saben. Es la típica pesadilla de un monstruo creado que adquiere vida por sí mismo, y se muestra autónomo y superior? ${ }^{7}$. El problema es que se terminan por socavar otras formas de gobierno, otros modos de actuar y de ser humano que quedan marginalizados por la "verdad" que produce el mercado. Incluso se mina la democracia liberal y la autonomía kantiana del individuo.

\footnotetext{
5 Por ejemplo, en el caso del capital humano (información como mercancía), el aprendizaje tiene que ver con comprar una mercancía (educación y salud). Como el sujeto compra la información, esta tuvo que ser generada en el pasado. La comunicación es igual a un intercambio.

${ }^{6}$ En esta etapa del paradigma informacional, se empiezan a expandir los juegos bayesianos, la importancia del "conocimiento común" y su importancia para los equilibrios de Bayes-Nash.

7 Películas como Her de Spike Jonze o 2001: Odisea en el espacio de Stanley Kubrick están relacionadas con este problema de máquinas que se salen del control humano y adquieren autonomía.
} 
Rob Horning (2012), al notar el impacto de esta revolución informacional en la literatura de espías y detectives, observa que se deja de retratar a estos como superhéroes con capacidades histriónicas à la Sherlock Holmes, para pasar a ser retratados como agentes "inteligentes contra su voluntad". Agentes en los que circula información, pero no son capaces de comprender la totalidad del misterio. Horning termina haciendo una reflexión sobre lo que se juega en este marco informacional.

"La búsqueda de información en lugar de conocimiento hace que funcionemos menos como pensadores que como procesadores, computadoras personales ineficientes y de baja potencia. No somos los sujetos que saben cosas o que producen conocimiento intencionalmente; En cambio, somos medios de circulación: objetos a través de los cuales la información pasa con más o menos ruido en la señal. No solo nos convertimos en parte de una red, sino también de un circuito. Somos peones en un juego más grande, "una mosca atrapada en las ruedas dentadas", como lo expresa Vandassy, el narrador de Epitaph for a Spy" (Horning, 2012).

\section{Neoliberalismo y lo común}

En nuestros días, pocos académicos gustan de utilizar la palabra neoliberalismo y pocas personas se definen como neoliberales. Parece que es un vocablo desgastado y que no es muy preciso. Sin embargo, las discusiones contemporáneas sobre el neoliberalismo son bastante pertinentes ${ }^{8}$. En el curso El nacimiento de la biopolítica que Foucault dicto en el College de France, observo diferencias fundamentales entre la argumentación de los autores liberales clásicos por un lado y el ordo-liberalismo alemán y la escuela de chicago por el otro (Foucault, 2004). Leyendo entre otros a Hayek y a Becker, Foucault nota que en estos autores hay "un arte de gobernar", una "reprogramación del gobierno liberal" que tiende a la necesidad de resolver los conflictos que se presentan en la sociedad. El neoliberalismo es así una racionalidad que tiende a estructurar y organizar las formas de vida a partir de la norma de competencia (Laval \& Dardot, 2013). Se trata de toda una "reconstrucción del alma". Por consiguiente, el neoliberalismo no es ni una ideología, ni una política económica. El neoliberalismo está presente sin que medien relaciones monetarias. Por consiguiente, el neoliberalismo es productor de relaciones sociales y de subjetividades. Foucault mostro como la teoría se filtra para moldear la razón y la práctica política. Así, no se trata solo de laissez faire, laissez passe. La discusión de las políticas neoliberales no es la del Estado versus el mercado, sino la de qué tipo de intervención realiza el Estado y para qué. Este marco cognitivo para entender el neoliberalismo es bastante pertinente ${ }^{9}$ por qué puede entender la diversidad de las dimensiones y practicas neoliberales que no son homogéneas ni en espacio ni en tiempo. Es claro por ejemplo que las políticas que propician mayor competencia en los mercados se instauraron en algunos países latinoamericanos de la mano de dictaduras, pero en otros países de forma suave, de la mano de gobernantes elegidos por el pueblo. El neoliberalismo ha utilizado la dictadura, la democracia o el endeudamiento para gobernar.

\footnotetext{
8 Se puede citar por ejemplo el fructífero debate que ocurrió hace unos pocos años en INET con autores como Philip Mirowski, Kari Polanyi Levitt, Matías Vernengo etc.

9 Por supuesto como señala Wendy Brown (2016), el planteamiento Foucaltiano también es problemático, por ejemplo, por su concepción de lo político, su análisis del origen del homo economicus, en no ver el capital como una relación de domino y en invisibilizar los efectos del neoliberalismo sobre la democracia y su imaginario (Brown, 2016).
} 
Desde este marco cognitivo, el neoliberalismo en Foucault tiene varios elementos importantes (Brown, 2016). En primer lugar, la competencia no es algo natural, sino que hay que producirla desde afuera y el estado tiene gran responsabilidad en producirla. En segundo lugar, se pretende la economización del estado y las políticas sociales. Así, el estado y la política social no deben desmercantilizar, ni reasignar la asignación del estado (ver la segunda sección), sino que deben promover la competencia. Así, "el estado asegura, defiende y apoya la economía". En tercer lugar, como eje articulador la competencia reemplaza al intercambio y con esto, la desigualdad reemplaza a la igualdad, el capital humano a la mano de obra y el emprendimiento a la producción. En cuarto lugar, ya no es el individuo el eje de la verdad. El mercado produce verdad pues sabe lo que los individuos deben hacer. Como vimos, este es uno de los ejes fundamentales de la economía informacional. En quinto lugar, se responsabiliza al Estado. El estado debe apoyar la economía, pero no debe intervenir ni en el nivel de intercambio, de la distribución, ni en los daños colaterales. Por último, el consenso político reemplaza la individuación y los conflictos políticos. "Todos esos socios de la economía, en la medida misma en que aceptan el juego económico de la libertad, producen un consenso que es de carácter político" (Foucault, 2004, pág. 106)

En resumen, el neoliberalismo articula "la competencia como norma de conducta y la empresa como modelo de subjetivación" (Laval \& Dardot, 2013). Se trata entonces de conducir o guiar las conductas de las personas en diferentes ámbitos de la vida. En la vida cotidiana puedo por ejemplo competir con mi pareja, sin ni siquiera ser consciente que lo hago, pues puede presentárseme como la lógica de la vida misma. El neoliberalismo tiende así a producir un auto-gobierno, una relación del yo consigo mismo bajo este tipo de relacionamiento competitivo, lo que Foucault llama el gobierno de sí y hacer de este el punto de aplicación del gobierno de los otros (Foucault, 2004). Desde este punto de vista, la revolución informacional presentada en el anterior capitulo es profundamente neoliberal, pues socaba la autonomía del individuo y del pueblo a favor de un mecanismo que profundiza la competencia.

Frente a esta racionalidad neoliberal, se trata de articular una racionalidad que pueda producir otras relaciones sociales, otras subjetividades; otro "gobierno de sí y de los otros". El principio de lo común es un "principio de coobligación (participación e implicación) para todos aquellos que están comprometidos en una actividad" (Laval \& Dardot, 2015). Lo común es una alternativa política y una racionalidad que pretende articular las contra conductas hacia la racionalidad neoliberal, en una nueva forma de racionalidad. Es decir, forjar y darle espacio a otro "arte de gobernar". Este principio ha nacido de las reivindicaciones de los movimientos sociales (por la democracia real, los movimientos en las plazas, por la educación, por el agua) que han visto recientemente una oleada de privatizaciones y "cerramientos" de bienes que son vitales para vivir una vida vivible. Es así una forma política de resistencia que han encarnado estos movimientos, pero también algo que busca la superación del capitalismo. Lo común es entonces un principio que articula la participación y la implicación en una actividad. Involucra sujetos activos en el establecimiento de las reglas que van a determinar una actividad como en su puesta en marcha; "co-participación y co-implicación".

Esta conceptualización diferencia entre el principio político de lo común, que se aplica, y los comunes, que se instituyen (Laval \& Dardot, 2015). Este es un marco cognitivo mucho 
más interesante para pensar los bienes comunes, pues en el marco neoclásico, estos bienes son definidos con referencia a unas propiedades que intrínsecamente tienen los objetos; esto es que los bienes sean no excluyentes pero rivales. Desde el marco cognitivo neoclásico es limitado el alcance de lo común, pues limita los comunes a un principio exterior y no como resultado de un proceso instituyente. Desde esta perspectiva, las empresas pueden ser comunes, como de hecho sucedió en las fábricas de recuperación en argentina desde 2001, en la que los trabajadores estaban participando e implicándose en la gestión y producción de la empresa. Todos los trabajadores participan en las decisiones sobre lo que hay que hacer y se implican en la puesta en obra. Esto por supuesto no podría suceder bajo el marco neoclásico, en la que las empresas se diferencian del mercado porque tienen una lógica jerárquica (Coase, 1988). Por contraste, desde el marco cognitivo de lo común, el mercado aparece como jerárquico, mientras que los comunes es el espacio de la democracia participativa.

El marco cognitivo entonces es esencial hoy para pensar nuestra vida social e individual. Allí donde el neoliberalismo ve la competencia como lazo social fundamental, lo común, produce otra relacionalidad con las cosas y con los seres vivos.

\section{Informalidad o economía popular.}

Tradicionalmente, la informalidad laboral se ha entendido como las actividades productivas que se realizan parcial o totalmente por fuera de la normativa que imponen los estados. El marco neoclásico en el que se suele interpretar este fenómeno, suele ver que la informalidad se produce como una respuesta óptima de los individuos ante cambios en los incentivos (Mejía \& Posada, 2007). En otros términos, son los cambios en la regulación lo que lleva a los individuos a la decisión de entrar o salir del mundo formal. Estos economistas suelen pensar que su análisis corresponde a la inteligibilidad misma del mundo social. Sin embargo, a esta forma de pensar le subyace una interpretación de que el mundo social está regido por un horizonte de individuos solitarios que actúan racionalmente ${ }^{10}$. Algunos análisis heterodoxos desafían este marco conceptual del individualismo metodológico para mostrar que la informalidad tiene sus causas en la estructura productiva de un país, sus patrones de especialización, su inserción en la economía internacional, los cambio en el mundo del trabajo y la empresa, la financiarización, etc. Estos análisis son muy pertinentes para desafiar la versión convencional del análisis dual del mercado laboral, desde la que se recomiendan políticas como la flexibilización del salario mínimo y la reducción de los costos laborales no salariales que desde su perspectiva tienden a expulsar a las personas al mercado informal.

Sin embargo, podría ser pertinente también cuestionar el mismo marco cognitivo de la dualidad ${ }^{11}$. Como argumenta Cesar Giraldo:

"El concepto de la informalidad induce a la connotación de que se trata de una realidad informe, sin estructura. De hecho, la literatura sobre la materia habla de "sector no estructurado". Sin embargo, es todo lo contrario: Al interior de la

\footnotetext{
10 No obstante, es claro que el mundo también puede leerse desde macrogrupos sociales o clases, que conllevan otra interpretación del mundo.

11 Hay varias versiones de la economía dual. "Economía pre capitalista versus capitalista, tradicional versus la moderna, marginal versus economía central, ejército de reserva versus asalariados, excluidos versus incluidos, y por supuesto, economía informal versus la formal". (Giraldo, 2017)
} 
economía popular existen estructuras y normas propias que la regulan, tales como reparto del territorio, mecanismos de financiación (por ejemplo, el gota a gota en Colombia), división del trabajo, horarios, sistemas de protección social, liderazgos. De manera que el llamado sector informal no es un sector informe, sino un sector estructurado en sus dimensiones económicas, sociales y políticas." (Giraldo, 2017, pág. 14).

Para dar cuenta de una realidad más amplia, ha emergido la categoría de economía de los sectores populares pretendiendo dar cuenta de las dimensiones micro que se quedan por fuera cuando se analiza bajo el binomio formal-informal. Este marco entonces disocia a-priori las relaciones entre economía popular y pobreza, entre economía popular y desorden, y entre economía popular y solidaridad.

Son entonces tres los marcos que se suspenden a-priori para dar mayor riqueza a los trabajos de campo y visualizar otra realidad. No se trata de negar la existencia de la pobreza o la solidaridad en estas economías, sino que estas no deben suponerse a-priori como regla o como marco.

Cambiar el encuadramiento permite transformar la manera en que se mira el problema. Desde esta perspectiva, el camino a la formalización ha sido un fracaso, ya sea por la vía del emprendimiento o de la salarización; los estudios de campo muestran que en estos sectores no hay aspiración de ser asalariado (Giraldo, 2017).

Se trata entonces, en primer lugar, de reconocer ${ }^{12}$ estas economías como un sector productivo que debe ser consultado y participe de la política, como se hace con otros gremios $^{13}$. Segundo, visualizar que "son ciudadanos a los que la sociedad no le retorna la compensación debida en cuanto a la valoración de su trabajo" (Giraldo, 2017, pág. 15). El problema del valor del trabajo cobra entonces toda su relevancia, pues se cuestiona el mismo criterio de valoración ya sea basado en la productividad, la tecnología o el valor agregado de la economía formal, para incluir otros como el trabajo social o los problemas y riesgos a los que está expuesto el trabajador popular. Parece difícil pensar así, pero es necesario efectuar la desnaturalización de los marcos cognitivos que damos por garantizados pues son invisibles cuando vivimos y pensamos.

Desde este encuadramiento el desafío es encontrar otros marcos de protección social distintos al del salariado y encontrar otras formas de valorizar el trabajo. La política pública en lugar de dirigirse a criminalizar a los vendedores en el espacio público, debe brindar los mecanismos para un mayor empoderamiento de estos sectores, como por ejemplo los créditos con bajas tasa de interés, en contravía de la solución de mercado de las microfinanzas.

En resumen, el marco de la economía popular permite un entendimiento diferente al binomio formal-informal, que, aunque pueda ser pertinente para pensar por ejemplo el cambio estructural, está basado en un orden normativo que no suele cuestionarse. Esta perspectiva brinda además la posibilidad de un verdadero dialogo y trabajo interdisciplinario.

\footnotetext{
12 Esto es fundamental para la discusión de ¿Quiénes tienen derecho a aparecer en el espacio público?
}

${ }^{13}$ En Colombia existen agrupaciones como el sindicato de trabajadores ambulantes entre otros. 


\section{Conclusiones: ¿Cómo conversar? Importancia de los marcos cognitivos para la heterodoxia}

Los debates en economía suelen dar por garantizado el horizonte significativo de los hechos. Se suele reclamar constantemente que "una cosa son los hechos y otra los valores" o que "los hechos son tozudos y hablan por sí mismos". Sin embargo, no debemos olvidar que los hechos no hablan por sí solos, siempre tiene que haber un observador que les de voz y esto se realiza dentro de un horizonte de significado. Este fue uno de los grandes descubrimientos de la fenomenología Husserliana y siglos atrás de las tradiciones orientales contemplativas. Esto es que la mente nunca puede quedar en blanco ${ }^{14}$ pues siempre se encuentra intencionada (se dirá en lenguaje fenomenológico, la conciencia siempre es conciencia de algo y está orientada a los objetos) (Husserl, 2014). Por consiguiente, la descripción de los objetos no se da sobre un plano neutro u objetivo. Siempre se narra o se cuenta una historia y toda descripción presupone al menos un relacionamiento con el mundo y con los objetos, una forma de ser-en-el-mundo como diría Heidegger.

En este articulo hemos visto como se cree estar debatiendo sobre lo que las cosas son en sí mismas. Sin embargo, el horizonte significativo de los hechos, nunca es intrínseco a los hechos mismos, sino que este posible por la intencionalidad de la conciencia que estructura inseparablemente la mente y el mundo (Husserl, 2014), lo que permite diferentes encuadramientos. La materialidad no tiene un significado intrínseco y esto nos lleva al problema, profundamente político, de las consecuencias que se pueden derivar de ello. Hoy por ejemplo una gran cantidad de discursos, entre los que se encuentra el coaching, la PNL y la psicología positiva entre otros, utilizan los marcos cognitivos para sustraer las reivindicaciones materiales, al encuadrar y enfatizar que los problemas son de actitud y de vencer al enemigo interior, con lo cual los marcos cognitivos son dispositivos de la racionalidad neoliberal.

No obstante, criticar este uso, no nos debe llevar a afirmar una imagen reduccionista del ser humano en el que se niegue la potencia de los marcos cognitivos. La mente no es solo un espejo o reflejo de la naturaleza y aunque puede comportarse de esta forma, no es su forma más propia u original. El uso y significado de los encuadramientos mentales es entonces un problema político y es posible mantener una posición crítica, a partir del uso que le damos a los marcos cognitivos. Como lo hemos mostrado, no todos los marcos tienen los mismos alcances, ni posibilitan las mismas cosas, sino que permiten ver y crear diferentes realidades, con consecuencias profundamente divergentes para la sociedad y los seres humanos.

Sin embargo, es importante para quienes tienen posiciones críticas, notar si están siendo "críticos" por qué toman distancia dentro de un marco cognitivo o porque critican el mismo punto de partida del marco. Hoy son varias las personas que, teniendo posturas "críticas", han sido "capturados" por los giros o desviaciones dentro del paradigma tradicional. Como se mostró, es lo que pasa cuando se piensa y se discute en términos de fallos de mercado, de irracionalidad, de sesgos cognitivos, del mercado como procesador de información, de bienes no excluyentes pero rivales, o de informalidad.

${ }^{14}$ Cómo hoy lo pretenden los falsos gurús y mercaderes de las tradiciones orientales. 
Por supuesto, no es la intención del articulo profundizar en las discusiones aquí ofrecidas, sino mostrar la importancia de los encuadramientos cognitivos por las consecuencias que surgen, y que es importante no debatir con las reglas del marco cognitivo del oponente. En otros términos, siempre debemos estar atentos, para no pensar y narrar la realidad económica, desde el mismo punto que se quiere combatir.

\section{Referencias}

Arrow, K., \& Debreu, G. (1954). Existence of an equilibrium for a competitive economy. Econometrica, Volume 22, Issue 3, 265-290.

Beck, U., \& Beck-Gernsheim, E. (2003). La individualización: el individualismo institucionalizado y sus consecuencias sociales y políticas. Barcelona: Paidos.

Brown, W. (2016). El pueblo sin atributos. La revolución secreta del neoliberalismo. Barcelona: Malpaso.

Coase, R. (1988). The Firm, the Market, and the Law. Chicago: Chicago University Press.

Davis, J. B. (2011). Individuals and Identity in Economics . Cambridge: Cambridge University Press.

Foucault, M. (2004). El nacimiento de la biopolítica. Mexico: FCE.

Friedman, M. (1966). Essays in positive economics. Chicago: University of Chicago Press.

Gigerenzer, G. (2008). Decisiones Instintivas. Barcelona: Ariel.

Giraldo, C. (2017). La economía popula desde abajo. Bogotá: Desde Abajo.

Horning, R. (18 de June de 2012). Agents without agency. Obtenido de New Inquiry: https://thenewinquiry.com/agents-without-agency/

Husserl, E. (2014). Ideas relativas a una fenomenología pura y una filosofía fenomenológica. México: FCE.

Kahneman, D. (2003). Maps of Bounded Rationality: Psychology for Behavioral economics (Nobel Speech). American Economic Review, Vol 93, No. 5, 1449-75.

Kahneman, D., Slovic, P., \& Tversky, A. (1982). Judgment under uncertainty: Heuristics and biases. Cambridge: Cambridge University Press.

Lakoff, G. (2008). Puntos de reflexion. Manual del progresista. Madrid: Peninsula.

Lakoff, G. (2009). Las metaforas de la vida cotidiana. Madrid: Catedra.

Laval, C., \& Dardot, P. (2013). La nueva razón del mundo. Ensayo sobre la sociedad neoliberal. Barcelona: Gedisa.

Laval, C., \& Dardot, P. (2015). Común. Ensayo sobre la revolución en el siglo XXI. Madrid: Gedisa.

Lawson, T. (1997). Economics and reality. London \& New York: Routledge.

Mankiw, G., \& Romer, D. (1991). New Keynessians Economics. Vol. 1: Imperfect Competition and Sticky Prices. Cambridge: The Mit Press .

Mantel, R. (1974). On the characterization of aggregate excess-demand. Journal of Economic Theory. 7, 348-353.

Mazzucato, M. (2014). El Estado Emprendedor. Madrid: RBA. 
Mazzucato, M. (2016). The Value Of Everything. Making and Taking in the Global Economy. New York: Public Affairs.

Mejía, D., \& Posada, C. (2007). Informalidad: teoría e implicaciones de política. Bogotá: Unidad de investigciones Banco de la República.

Minsky, H. (1982). Can It Happen Again?: Essays on Instability and Finance. New York: Routledge.

Minsky, H. (1990). Las razones de Keynes. Mexico: FCE.

Mirowski, P., \& Nik-Khah, E. (2017). The knowledge we have lost with information. New York: Oxford University Press.

Negri, T., \& Hardt, M. (2011). Commonwealth. Madrid: Akal.

Pfister, H.-R., \& Böhm, G. (2008). The multiplicity of emotions: A framework of emotional functions in decision making. Judgment and Decision Making, 3, 5-17.

Primrose, D. (2017). The Subjectification of Homo Economicus in Behavioural Economics. Journal of Australian Political Economy, 88-128.

Raworth, K. (2017). Doughnut Economics. Seven ways to think like a 21st century economist. Oxford: Oxford University Press.

Rodrick, D. (2016). Las leyes de la economía. Los aciertos y errores de una ciencia en entredicho. Barcelona: Deusto.

Samuelson, P. (1957). Fundamentos del analisis económico. Buenos Aires: Ateneo.

Sen, A. (1977). Rational Fools. A Critique of the Behavioral Foundations of Economic Theory. Philosophy \& Public Affairs, Vol. 6, No. 4, 317-344.

Sonnenschein, H. (1972). Market excess-demand functions. Econometrica 40 (3), 549-563.

Starr, R. (2013). Why is there Money? Walrasian general equilibrium foundations of monetary theory. Cheltenham: Edward Elgar.

Stiglitz, J., Ocampo, J. A., Pfrench-Davis, R., Spiegel, S., \& Nayyar, D. (2006). Stability with Growth: Macroeconomics, Liberalization and Development. Oxford: Oxford University Press.

Thaler, R., \& Sunstein, C. (2008). Nudge: Improving Decisions about Health, Wealth,. New Haven: Yale University Press.

Varela, F. (2005). Conocer. Las ciencias cognitivas: tendencias y perspectivas. Cartografía de las ideas actuales. Barcelona: Gedisa.

Wang, X. T., Simons, F., \& Brédart, S. (2001). Social cues and verbal framing in risky choice. Journal of Behavioral Decision Making, 14(1), 1-15. 